\title{
Feature quarrels: The Dempster-Shafer Evidence Theory for Image Segmentation Using a Variational Framework *
}

\author{
Björn Scheuermann and Bodo Rosenhahn \\ Institut für Informationsverarbeitung \\ Leibniz Universität Hannover, Germany \\ \{scheuermann, rosenhahn\}@tnt. uni-hannover.de
}

\begin{abstract}
Image segmentation is the process of partitioning an image into at least two regions. Usually, active contours or level set based image segmentation methods combine different feature channels, arising from the color distribution, texture or scale information, in an energy minimization approach. In this paper, we integrate the Dempster-Shafer evidence theory in level set based image segmentation to fuse the information (and resolve conflicts) arising from different feature channels. They are further combined with a smoothing term and applied to the signed distance function of an evolving contour. In several experiments we demonstrate the properties and advantages of using the DempsterShafer evidence theory in level set based image segmentation.
\end{abstract}

\section{Introduction}

Image segmentation is a popular problem in the field of computer vision. It is the first step in many fundamental tasks which pattern recognition and computer vision have to deal with. The problem has been formalized by Mumford and Shah as the minimization of a functional [1]. Level set representations or active contours [2-4] are a very efficient way to find minimizers of such a functional.

As shown by many papers and textbooks on segmentation [2-8], a lot of progress has been made using these variational frameworks. However it still faces several difficulties which are usually caused by violations of model assumptions. For example, homogeneous [2] or smooth [1] object regions are usually assumed. Due to noise, occlusion, texture or shading this is often not appropriate to delineate object regions. Statistical modeling of regions [5] and supplement of additional information such as texture [6], motion [7] or shape priors [8] increases the number of scenes where image segmentation using variational frameworks succeeds.

Another reason for failed segmentations is a unsuitable or false initialization, which is necessary to find a local minimizer for a segmentation functional.

Earlier works on image segmentation using the Dempster-Shafer theory of evidence have been presented in [9-12] and in [13]. These works combined the

* This work is partially funded by the German Research Foundation (RO 2497/6-1). 


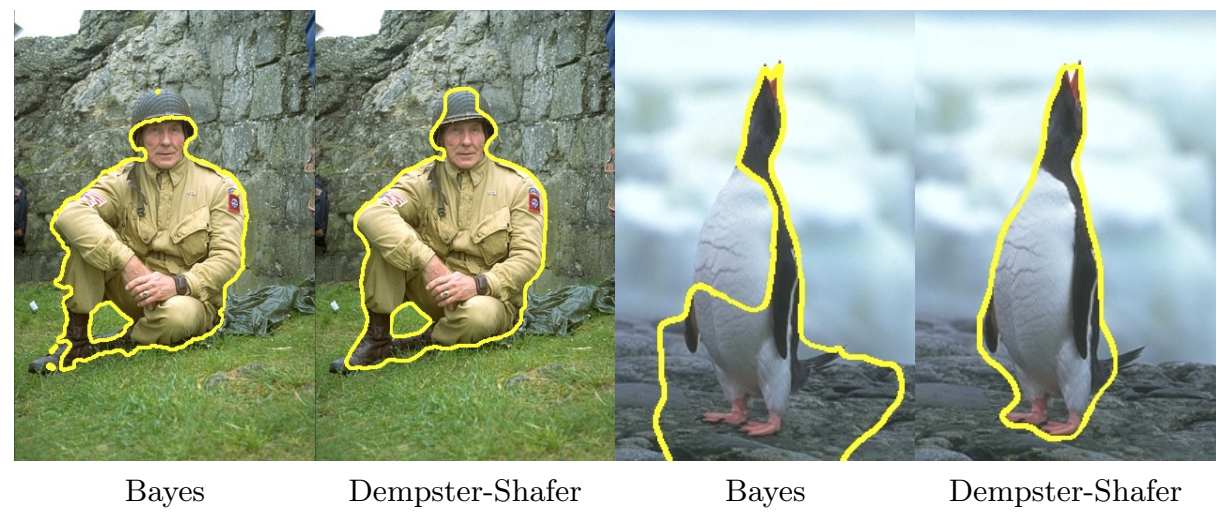

Fig. 1: Segmentation results using either the probabilistic Bayesian model or Dempster-Shafer evidence theory to fuse information.

evidence theory with either a simple threshholding [9], a decisional procedure [10], a fuzzy clustering algorithm [11], a region merging algorithm [12] or a $k$-means clustering algorithm [13]. All cited works use the idea of DempsterShafer to fuse the informations arising from three color channels. In contrast to these works we propose to combine the evidence theory with a variational segmentation framework, including statistical modeling of regions, the respective Euler-Lagrange equations and a smoothness term.

\subsection{Contribution}

In this paper we propose to use the Dempster-Shafer theory of evidence [14, 15] to combine several information sources in a variational segmentation framework. This theory of evidence is an elegant way to fuse information from different feature channels, e.g., color channels and texture information, and it offers an alternative to traditional probabilistic theory. Instead of using the probabilistic Bayesian model to fuse separated probability densities we deal with the Dempster-Shafer evidence theory which allows to represent inaccuracy and uncertainty at the same time. A prerequisite for the Dempster-Shafer theory of evidence is a mass function. We show how to define these functions and combine them to derive the so called belief function. This belief function represents the total belief for a hypothesis and can be integrated into a variational segmentation framework to assign more support to high probabilities (see Figure 1).

Note, that image segmentation is just our example application, but we strongly believe that the Dempster-Shafer evidence theory can be advantageous for other applications in Computer Vision or Machine Learning as well. Therefore, this theory is of general interest for our research community. 


\subsection{Paper Organization}

In Section 2 we continue with a review of the variational approach for image segmentation, which is the basis for our segmentation framework, and a presentation of the Dempster-Shafer theory of evidence. Section 3 introduces the proposed segmentation method which combines the variational segmentation method and the Dempster-Shafer theory of evidence. Experimental results in Section 4 demonstrate the advantages of the proposed method. The paper finishes with a short conclusion.

\section{Background}

\subsection{Image Segmentation using a Variational Framework}

Our variational segmentation framework is based on the works of $[2,16]$. Using the level set formulation for the general problem of image segmentation has several well known advantages, e.g. the naturally given possibility to handle topological changes of the 1-D boundary curve. This is especially important if the object is partially occluded by another object or if the object consist of multiple parts.

In case of a two-phase segmentation, the level set function $\varphi: \Omega \rightarrow \mathbb{R}$ splits the image domain $\Omega$ into two regions $\Omega_{1}, \Omega_{2} \subseteq \Omega$ with

$$
\varphi(x)=\left\{\begin{array}{l}
\geq 0, \text { if } x \in \Omega_{1} \\
<0, \text { if } x \in \Omega_{2}
\end{array} .\right.
$$

The zero-level line of the function $\varphi(x)$ represents the boundary between the object, which is sought to be extracted, and the background. A successful remedy to extend the number of situations in which image segmentation can succeed is the use of additional constraints, for instance the restriction to a certain object shape $[17,18]$. However in this work we focus on segmentation without using shape priors.

The basis for our segmentation framework is the so called Chan-Vese energy functional [2]:

$$
\begin{aligned}
E(\varphi)= & -\int_{\Omega}\left(H(\varphi) \log p_{1}+(1-H(\varphi)) \log p_{2}\right) d \Omega \\
& +\nu \int_{\Omega}|\nabla H(\varphi)| d \Omega,
\end{aligned}
$$

where $\nu \geq 0$ is a weighting parameter between the given constraints, $p_{i}$ are probability densities and $H(s)$ is a regularized Heaviside function. The regularized Heaviside function is needed to build the Euler-Lagrange equation and to make it possible to indicate at each iteration step to which region a pixel belongs. Given the two probability densities $p_{1}$ and $p_{2}$ of $\Omega_{1}$ and $\Omega_{2}$ the total a-posteriori probability is maximized by minimizing the first term, i.e. pixels are assigned to 
the most probable region according to the Bayes rule. The second term penalizes the length of the contour and act as a smoothing term.

Minimization of the Chan-Vese energy functional (2) can be easily performed by solving the corresponding Euler-Lagrange equation to $\varphi$. This leads to the following partial differential equation

$$
\frac{\partial \varphi}{\partial t}=\delta(\varphi)\left(\log \frac{p_{1}}{p_{2}}+\nu \operatorname{div}\left(\frac{\nabla \varphi}{|\nabla \varphi|}\right)\right),
$$

where $\delta(s)$ describes the derivative of $H(s)$ with respect to its argument. Starting with some initial contour $\varphi^{0}$ and given the probability densities $p_{1}$ and $p_{2}$ one has to solve the following initial value problem

$$
\left\{\begin{array}{l}
\varphi(x, 0)=\varphi^{0} \text { for } x \in \Omega \\
\frac{\partial \varphi}{\partial t}=\delta(\varphi)\left(\log \frac{p_{1}}{p_{2}}+\nu \operatorname{div}\left(\frac{\nabla \varphi}{|\nabla \varphi|}\right)\right)
\end{array}\right.
$$

Thus, the quality of the segmentation process is limited by the initial contour and the way the foreground and background probability densities $p_{1}$ and $p_{2}$ are modeled. In this paper we used the nonparametric Parzen estimates [6], which is a well known histogram-based method. This Model was chosen since compared to multivariate Gaussian Mixture Models (GMM) it leads to comparable results without estimating so many parameters. Other possibilities to model the probability densities given the image cues are, e.g., a Gaussian density with fixed standard deviation [2] or a generalized Laplacian [19].

For the proposed method it is necessary to extend this segmentation framework from gray scale images to feature vector images $I=\left(I_{1}, \ldots, I_{m}\right)$. This extension is straight forward and has been applied to the Chan-Vese model [20]. For this extension it is assumed that the channels $I_{j}$ are independent, so that the total a posteriori probability density $p_{i}(I)$ of region $\Omega_{i}$ is the product of the separated probability densities $p_{i, j}\left(I_{j}\right)$. Thus the Chan-Vese model (2) for vector images reads:

$$
\begin{aligned}
E(\varphi)= & -\int_{\Omega} H(\varphi) \sum_{j=1}^{m} \log p_{1, j} d \Omega-\int_{\Omega}(1-H(\varphi)) \sum_{j=1}^{m} \log p_{2, j} d \Omega \\
& +\nu \int_{\Omega}|\nabla H(\varphi)| d \Omega
\end{aligned}
$$

and yields to the following Euler-Lagrange equation:

$$
\frac{\partial \varphi}{\partial t}=\delta(\varphi)\left[\sum_{j=1}^{m} \log \frac{p_{1, j}}{p_{2, j}}+\nu \operatorname{div}\left(\frac{\nabla \varphi}{|\nabla \varphi|}\right)\right] .
$$

Because of their independency, the $p_{i, j}$ can be estimated for each region $i$ and each channel $j$ separately. In other words, for each pixel in the image the foreground probability $\sum_{j=1}^{m} \log p_{1, j}$ and the background probability $\sum_{j=1}^{m} \log p_{2, j}$ is computed over all feature channels $j$. Possible image features to be incorporated by means of this model are color, texture $[21,6]$ and motion [7]. 


\subsection{Dempster-Shafer evidence theory}

The Dempster-Shafer evidence theory, also called evidence theory, was first introduced in the late 60s by A.P. Dempster [14], and formalized in 1976 by G. Shafer [15].

This theory is often described as a generalization of the Bayesian theory to represent inaccuracy and uncertainty information at the same time. The basic idea of the evidence theory is to define a so called mass function on a hypotheses set $\Omega$, also called frame of discernment. Let us note the hypotheses set $\Omega$ composed of $n$ single mutually exclusive subsets $\Omega_{i}$, which is symbolized by:

$$
\Omega=\left\{\Omega_{1}, \Omega_{2}, \ldots, \Omega_{n}\right\} .
$$

In order to express a degree of confidence for each element $A$ of the power set $\wp(\Omega)$, it is possible to associate an elementary mass function $m(A)$ which indicates all confidences assigned to this proposition. The mass function $m$ is defined by:

$$
m: \wp(\Omega) \rightarrow[0,1]
$$

For all hypotheses, $m$ must fulfill the following conditions:

$$
\text { (i) } m(\emptyset)=0 \quad \text { (ii) } \quad \sum_{A_{n} \subseteq \Omega} m\left(A_{n}\right)=1 .
$$

In this context, the quantity $m(A)$ can be interpreted as the belief strictly placed on hypothesis $A$. This quantity differs from a Bayesian probability function by the totality of the belief which is distributed not only on the simple classes, but also on the composed classes. This modeling shows the impossibility to dissociate several hypotheses. This characteristic is the principal advantage of the evidence theory, but it also represents the principal difficulty of this method.

If $m(A)>0$, hypothesis $A$ is a so called focal element. The union of all the focal elements of a mass function is called the core $N$ of the mass function

$$
N=\{A \in \wp(\Omega) \mid m(A)>0\} .
$$

From the basic belief assignment $m$, a belief function $\mathrm{Bel}: \wp(\Omega) \rightarrow[0,1]$ and a plausibility function $P l: \wp(\Omega) \rightarrow[0,1]$ can be defined as

$$
\operatorname{Bel}(A)=\sum_{A_{n} \subseteq A} m\left(A_{n}\right), \quad P l(A)=\sum_{A \cap A_{n} \neq \emptyset} m\left(A_{n}\right),
$$

with $A_{n} \in \wp(\Omega)$. $\operatorname{Bel}(A)$ is interpreted as the total belief committed to hypothesis $A$, that is, the mass of $A$ itself plus the mass attached to all subsets of $A$. $\operatorname{Bel}(A)$ then is the total positive effect the body of evidence has on a value being in $A$. It quantifies the minimal degree of belief of the hypothesis $A$.

A particular characteristic of the Dempster-Shafer evidence theory (one which makes it different from probability theory) is that if $\operatorname{Bel}(A)<1$, then the remaining evidence $1-\operatorname{Bel}(A)$ needs not necessarily refute $A$ (i.e., support its negation 
$\bar{A})$. That is, we do not have the so-called additivity rule $\operatorname{Bel}(A)+\operatorname{Bel}(\bar{A})=1$. Some of the remaining evidence may be assigned to propositions which are not disjoint from $A$, and hence could be plausibly transferable to $A$ in the light of new information. This is formally represented by the plausibility function $\mathrm{Pl}$ (see Eq. (11)). $P l(A)$ is the mass of hypothesis $A$ and the mass of all sets which intersect with $A$, i.e. those sets which might transfer their mass to $A . P l(A)$ is the extent to which the available evidence fails to refute $A$. It quantifies the maximal degree of belief of hypothesis $A$.

The relation between mass function, belief function and plausibility function is described by:

$$
m(A) \leq \operatorname{Bel}(A) \leq \operatorname{Pl}(A) \quad \forall A \in \wp(\Omega)
$$

Dempster's rule of combination The Dempster-Shafer theory has an important operation, the Dempster's rule of combination, for pooling of evidence from a variety of features. This rule aggregates two independent bodies of evidence defined within the same frame of discernment into one body of evidence. Let $m_{1}$ and $m_{2}$ be two mass functions associated to two independent bodies of evidence defined in the same frame of discernment. The new body of evidence is defined by

$$
m(A)=m_{1}(A) \otimes m_{2}(A)=\frac{\sum_{B \cap C=A} m_{1}(B) m_{2}(C)}{1-\sum_{B \cap C=\emptyset} m_{1}(B) m_{2}(C)} .
$$

Dempster's rule of combination computes a measure of agreement between two bodies of evidence concerning various propositions discerned from a common frame of discernment. Since Dempster's rule of combination is associative, we can combine information coming from more than two feature channels.

\section{Dempster-Shafer evidence theory for variational image segmentation}

To introduce the proposed method, using the Dempster-Shafer evidence theory to fuse information arising from different feature channels, we consider the following example. Let $I=\left(I_{1}, I_{2}\right)$ be a vector image with two feature channels and $\varphi^{t}$ with $t \geq 0$ be a contour dividing the image into foreground and background. To minimize the energy functional (5), foreground and background probabilities need to be computed over both feature channels. Using the Bayesian model and disregard the smoothing term, a pixel is defined as foreground if

$$
\sum_{i=1}^{2} \log \left(\frac{p_{1, i}}{p_{2, i}}\right)>0 \text {. }
$$

Our proposed method uses the Dempster-Shafer theory of evidence to fuse the information coming from the feature channels. Therefore, we have to define the 


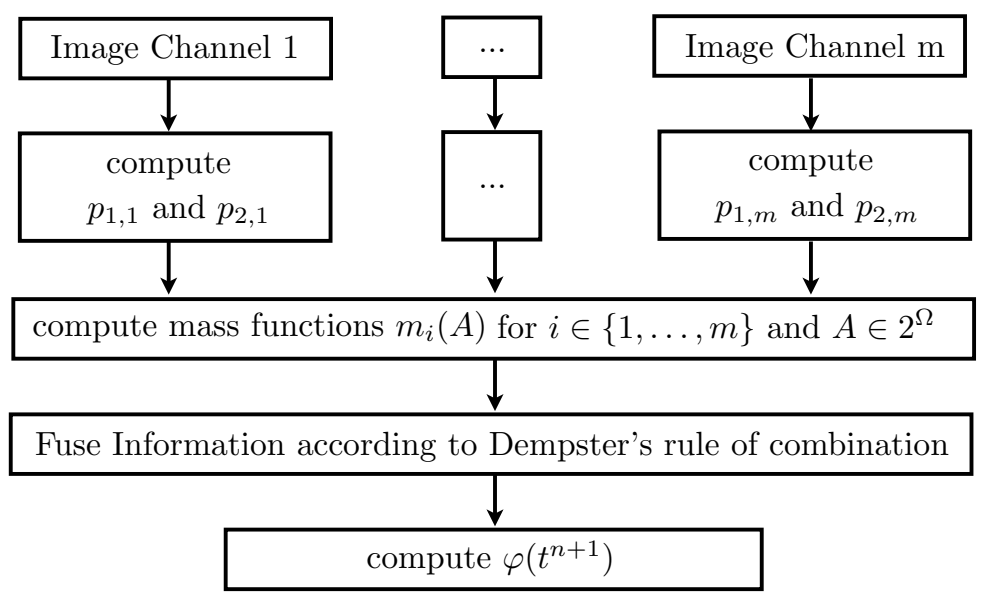

Fig. 2: Proposed image segmentation paradigm based on combining the variational framework and Dempster-Shafer theory of evidence.

mass function $m: \wp(\Omega) \rightarrow[0,1]$. In case of a two-phase segmentation, the frame of discernment becomes $\Omega=\left(\Omega_{1}, \Omega_{2}\right)$ where $\Omega_{1}$ denotes the object or foreground, and $\Omega_{2}$ the background. We propose to define the mass function $m_{j}$ for all hypotheses as:

$$
\begin{aligned}
& m_{j}\left(\Omega_{1}\right)=p_{1, j}(I(x)), \quad m_{j}\left(\Omega_{2}\right)=p_{2, j}(I(x)), \\
& \left.m_{j}(\emptyset)=0, \quad m_{j}(\Omega)=1-\left(p_{1, j}(I(x))\right)+p_{2, j}(I(x))\right),
\end{aligned}
$$

for $j \in\{1,2\}$. In practice, the defined mass functions fulfill Eq. (9). If $p_{1, j}+p_{2, j}>$ 1 normalization is straight forward to fulfill the condition. Dempster's rule of combination (Eq. (13)) fuses the two bodies of evidence to compute a measure of agreement between both mass functions. We obtain the new mass function $m=m_{1} \otimes m_{2}$, with Dempster's rule of combination (13).

In case of a two-phase segmentation, the total belief committed to a focal element $\Omega_{i}$ is equal to the belief strictly placed on $\Omega_{i}$. Thus we obtain

$$
\operatorname{Bel}\left(\Omega_{i}\right)=m\left(\Omega_{i}\right) \text { for } i \in\{1,2\} \text { and } \operatorname{Bel}(\Omega)=1 .
$$

If we use the total belief committed to a focal element and disregard the smoothing term, a pixel is defined as foreground if

$$
\log \left(\frac{\operatorname{Bel}\left(\Omega_{1}\right)}{\operatorname{Bel}\left(\Omega_{2}\right)}\right)>0
$$

The plausibility function $\operatorname{Pl}\left(\Omega_{i}\right)$, which quantifies the maximal degree of belief of the hypothesis $\Omega_{i}$ defines a pixel as foreground if

$$
\log \left(\frac{P l\left(\Omega_{1}\right)}{P l\left(\Omega_{2}\right)}\right)=\log \left(\frac{m\left(\Omega_{1}\right)+m(\Omega)}{m\left(\Omega_{2}\right)+m(\Omega)}\right)>0 .
$$




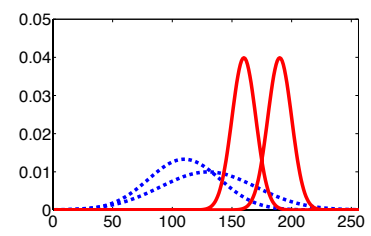

(a)

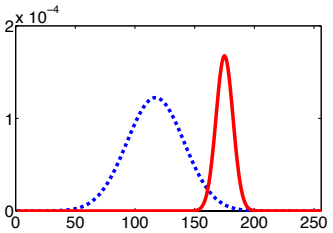

(b)

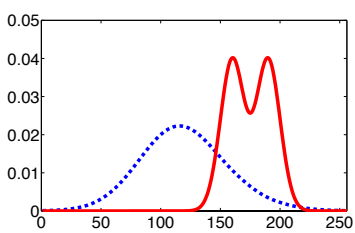

(c)

Fig. 3: (a) Illustration of two foreground probability densities (solid red line) and two background probability densities (dashed blue line), (b) product of the two separated densities (Bayesian model), (c) mass functions (given by Dempster's rule of combination (13)); Using the evidence theory to fuse the densities enlarges the area of the supported foreground

Our proposed method uses the total belief committed to foreground or background region. Figure 2 shows the general segmentation paradigm to evolve a contour combining the variational framework and Dempster-Shafer evidence theory.

In general, let $I=\left(I_{1}, \ldots, I_{k}\right)$ be a vector image with $k$ feature channels. Assuming independent feature channels and using the Bayesian model, the total a-posteriori probability of a region is the product of the separated probability densities. With Dempster-Shafer's theory of evidence, the information arising from the $k$ feature channels is fused using Dempster's rule of combination, resulting in:

$$
m=m_{1} \otimes m_{2} \otimes \ldots \otimes m_{k},
$$

where $m_{j}$ is defined by Equation (9). Now, the total belief committed to a region is used as the a-posteriori probability of a region. Thus, the new energyfunctional of our proposed method is given by:

$$
\begin{aligned}
E(\varphi)= & -\int_{\Omega} H(\varphi) \log \operatorname{Bel}\left(\Omega_{1}\right) d \Omega-\int_{\Omega}(1-H(\varphi)) \log \operatorname{Bel}\left(\Omega_{2}\right) d \Omega \\
& +\nu \int_{\Omega}|\nabla H(\varphi)| d \Omega
\end{aligned}
$$

and yields to the following Euler-Lagrange equation:

$$
\frac{\partial \varphi}{\partial t}=\delta(\varphi)\left[\log \frac{\operatorname{Bel}\left(\Omega_{1}\right)}{\operatorname{Bel}\left(\Omega_{2}\right)}+\nu \operatorname{div}\left(\frac{\nabla \varphi}{|\nabla \varphi|}\right)\right] .
$$

Figure 3 shows the differences between both models and clarifies the effect of the new method. Given two foreground and two background probability densities in Figure 3a, the product of the separated probability densities is shown in Figure $3 \mathrm{~b}$ and the total belief function coming from Dempster's rule of combination 


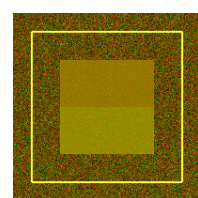

(a)

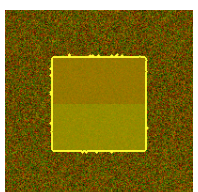

(b)

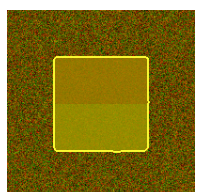

(c)

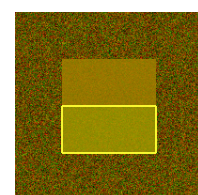

(d)

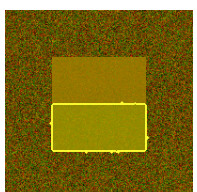

(e)

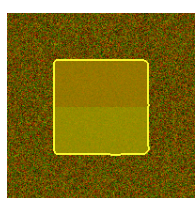

(f)

Fig. 4: (a) and (d): Two different initializations for the segmentation methods, (b) and (e): final segmentations using the Bayesian model, (c) and (f): final segmentations using Dempster-Shafer evidence theory. While the proposed method converges to almost identical results, the Bayesian model get stuck in two different local minima, due to one inadequate foreground histogram. Evaluating the energy of the results shows that the energy of (e) is the bigger than the other ones.

in Figure 3c. Obviously, the effect of the foreground probability in Figure 3c is larger than in Figure 3b, since the Dempster-Shafer theory of evidence supports high probabilities whereas the Bayesian model supports small probabilities.

Intuitively this means that the Bayesian model searches for feature channels not supporting a region whereas the Dempster-Shafer theory searches for feature channels that support a specific region.

Properties of the Bayesian model, e.g., to include shape priors [22], can be transferred straightforward to the proposed framework by adding terms to the new energy-functional (20).

\section{Experimental Results}

We introduced a segmentation method which integrates the Dempster-Shafer theory of evidence into a variational segmentation framework. To show the effect of the new approach, we present some results of our proposed method and compare them to segmentation results using the Bayesian framework to fuse information arising from different feature channels.

We evaluate our method on real images taken from the Berkeley segmentation dataset [23], our own real images, as well as on synthetic textured images from the Prague texture segmentation data-generator and benchmark [24]. In most of our experiments we used the CIELAB color channels and the smoothing term. This implies that we do not integrate additional information such as texture or shape priors in most of our experiments. To demonstrate the advantages of our framework we integrated texture features for the experiments on the synthetic textured images.

A situation in which the advantages of the proposed method become apparent is the synthetic example shown in Figure 4. While the segmentation using the Bayesian model computes different segmentation boundaries for the two initializations, the proposed methods converges to almost identical results for both 


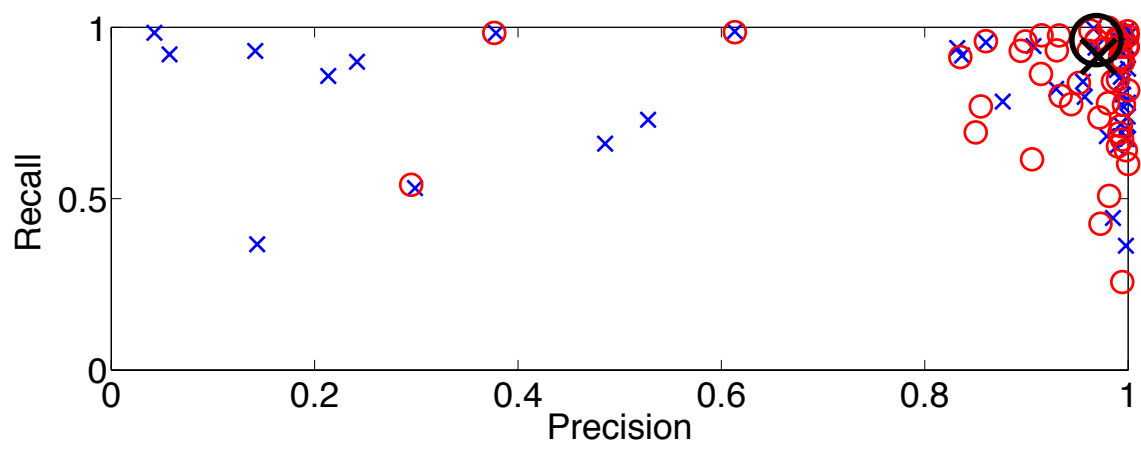

Fig. 5: Precision-Recall-Diagram. Circles mark the performance using DempsterShafer theory of evidence, $\mathrm{x}$ marks the performance using Bayes. The black marks mark the mean performance (Dempster-Shafer: 0.93, 0.83; Bayes: 0.81, 0.82).

initializations. The reason for the different segmentation results with the initialization in Figure 4d is a inadequate foreground statistic for one of the two feature channels (red and green). For the upper part of the object, that has to be segmented, this inadequate feature channel (green) supports the foreground with very small probabilities. More formally:

$$
\exists j \in\{1,2\} \mid p_{1, j}(x) \approx 0 \quad \forall x \in A,
$$

while $A$ is the upper part of the object. Using the Bayesian model we obtain

$$
p_{1}(x)=\prod_{j} p_{1, j}(x) \approx 0 .
$$

Because of the noisy background, these statistics have a larger standard deviation than the foreground statistics (see also Figure 3). For our example we obtain

$$
\begin{aligned}
& p_{1,1}(x) \in[0.024 ; 0.040], p_{1,2}(x) \approx 0, \\
& p_{2,1}(x) \in[0.006 ; 0.004], p_{2,2}(x) \in[0.007 ; 0.006],
\end{aligned}
$$

for $x \in A$, which results in

$$
p_{2}(x)=\prod_{j} p_{2, j}(x)>p_{1}(x) \quad \forall x \in A .
$$

Apparently the first feature channel (red) is not considered, even if it supports the foreground with the highest probability. With the Dempster-Shafer evidence theory this problem is solved elegantly since in the aforementioned case we have

$$
\operatorname{Bel}\left(\Omega_{1}\right)>\operatorname{Bel}\left(\Omega_{2}\right) \quad \forall x \in A,
$$


This may be explained by the fact, that the Dempster-Shafer evidence theory assigns more support to high probabilities whereas the Bayesian model more strongly supports small probabilities (see also Figure 3).

The same effect of the evidence theory can be observed in real images, where the Bayesian model does not segment parts of the object, because one feature channel is approximately zero (e.g. Figure 1, or the tent in Figure 7). Conversely, the proposed method converges to a good segmentation in these regions because the two other channels strongly support the foreground.

To evaluate and demonstrate the impact of the new method, we choose 47 images from the Berkeley segmentation dataset [23]. This subset was chosen for practical reasons, because most of the images are not suited for variational image segmentation. To measure the performance we calculated precision and recall for each of the images, which are defined by:

$$
\text { Precision }=\frac{|G \cap R|}{|G \cap R|+|R \backslash G|}, \quad \text { Recall }=\frac{|G \cap R|}{|G \cap R|+|G \backslash R|},
$$

where $G$ is the ground-truth foreground object and the region $R$ is the objectsegment derived from the segmentation. As ground-truth foreground we used the manual segmented ground-truth from [25], which is publicly available. For both frameworks we used the same manually selected initialization for each image, which was typically a rectangular inside the object. We decided to use manually segmentations, since both methods find local minimizers of the given energy functional. The regularization parameter was chosen slightly different between the frameworks but remained the same for all images. The probability densities where modeled as nonparametric Parzen estimates.

The performance of all images is shown in Figure 5. Using the Bayesian framework to fuse the information leads to a mean precision of 0.81 and a mean recall of 0.82 while the mean precision using the proposed Dempster-Shafer theory is 0.92 and the mean recall is 0.83 .

Figures 7 and 1 show some example segmentations on images from the Berkeley segmentation dataset. Using our proposed method to fuse the different information helps to segment and separate much better the semantically interesting and different regions by searching for features that support the foreground region or the background region. Analyzing the lower right Example of Figure 7 shows, that the Bayesian model segment parts of the swamp, because on channel does not support the background. Sometimes the proposed model leads to slightly worse segmentations. E.g. analyzing the flower, some parts of the blossoms are not segmented. The reason why the Bayesian model segments these parts is one channel that does not support the background.

Example segmentations integrating texture features are given in Figure 6. Again the proposed method separates the the interesting regions much better than the Bayesian approach. 


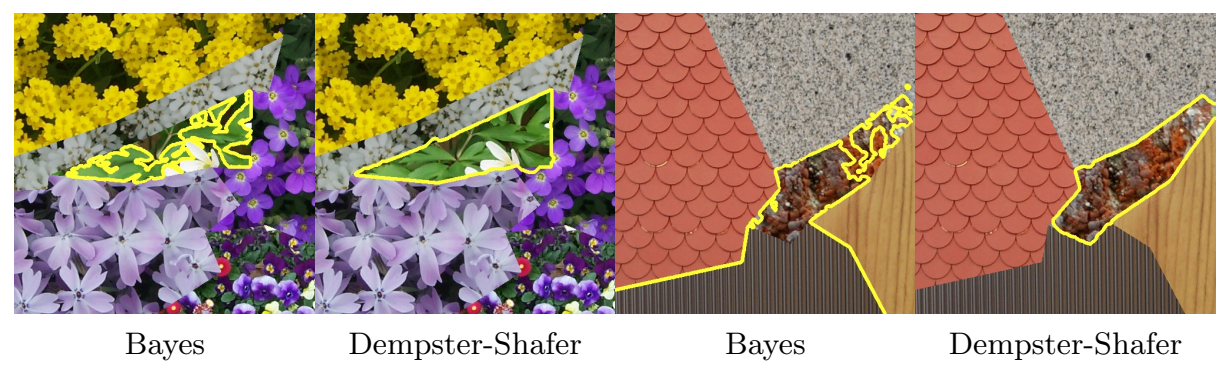

Fig. 6: Segmentation results using either the probabilistic Bayesian model or Dempster-Shafer evidence theory to fuse color and texture information.

\section{Conclusion}

In this paper we proposed the Dempster-Shafer evidence theory as an extension of the Bayesian model for the task of level set based image segmentation. The main property of this theory is to combine information arising from different feature channels by modeling inaccuracy and uncertainty at the same time. It therefore allows to fuse these information and resolve conflicts. In several experiments on real and synthetic images we demonstrated the properties and advantages of using the Dempster-Shafer evidence theory for image segmentation.

We strongly believe that this theory is of high interest for other applications in the fields of Computer Vision or Machine Learning as well.

\section{References}

1. Mumford, D., Shah, J.: Boundary detection by minimizing functionals. In: IEEE Computer Society Conference on Computer Vision and Pattern Recognition, San Francisco, CA, IEEE Computer Society Press, Springer (1985) 22-26

2. Chan, T., Vese, L.: Active contours without edges. IEEE Transactions on Image Processing 10 (2001) 266-277

3. Kass, M., Witkin, A., Terzopoulos, D.: Snakes: Active Contour models. International Journal of Computer Vision 1 (1988) 321-331

4. Osher, S., Paragios, N.: Geometric level set method in imaging, vision, and graphics. Springer-Verlag (2003)

5. Zhu, S.C., Yuille, A.: Region competition: unifying snakes, region growing, and bayes/mdl for multiband image segmentation. IEEE Transaction on Pattern Analysis and Machine Intelligence 18 (1996) 884-900

6. Rousson, M., Brox, T., Deriche, R.: Active unsupervised texture segmentation on a diffusion based feature space. In: IEEE Computer Society Conference on Computer Vision and Pattern Recognition, Madison, WI (2003) 699-704

7. Cremers, D., Soatto, S.: Motion competition: A variational approach to piecewise parametric motion segmentation. International Journal of Computer Vision 62 (2004) 249-265 

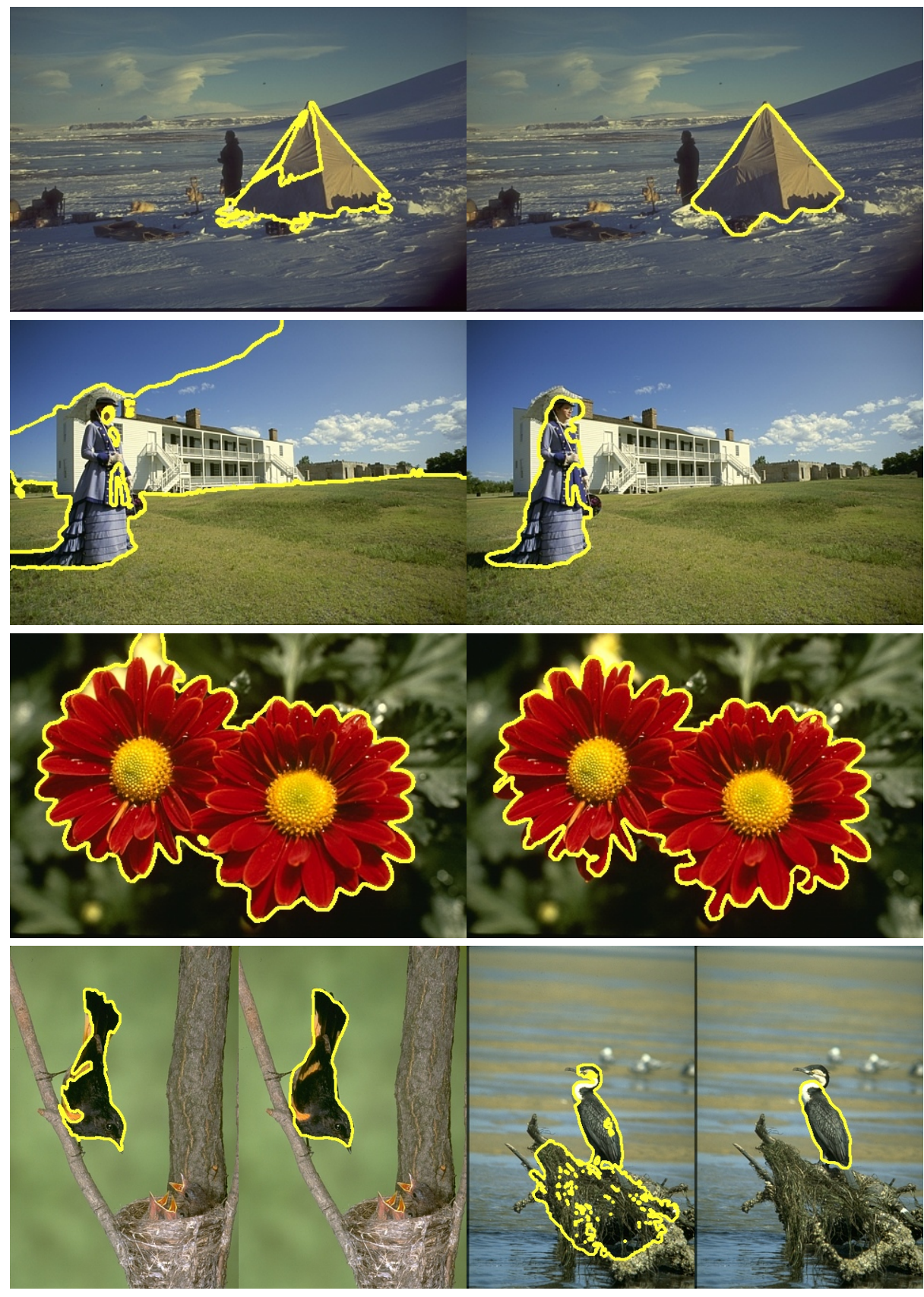

Fig. 7: Images taken from the Berkeley dataset [23]. The left image shows the segmentation results using the Bayesian model to fuse the CIELAB-color-channels, the right image shows the results using Dempster-Shafer theory of evidence to fuse the information. Each example shows that the evidence theory can help to improve the segmentation. 
8. Malladi, R., Sethian, J.A., Vemuri, B.C.: Shape modeling with front propagation: A level set approach. IEEE Transactions on Pattern Analysis and Machine Intelligence 17 (1995) 158-175

9. Mena, J.B., Malpica, J.A.: Color image segmentation using the dempster-shafer theory of evidence for the fusion. In: International Archives of the Photogrammetry, Remote Sensing and Spatial Information Sciences (34) 3/W8. (2003) 139-144

10. Chaabane, S., Sayadi, M., Fnaiech, F., Brassart, E.: Color image segmentation based on dempster-shafer evidence theory. In: 14th IEEE Mediterranean Electrotechnical Conference. (2008) 862-866

11. Chaabane, S.B., Sayadi, M., Fnaiech, F., Brassart, E.: Dempster-Shafer evidence theory for image segmentation: application in cells images. International Journal of Signal Processing (2009)

12. Adamek, T., O'Connor, N.E.: Using Dempster-Shafer Theory to Fuse Multiple Information Sources in Region-Based Segmentation. In: 2007 IEEE International Conference on Image Processing, IEEE (2007) 269 - 272

13. Rombaut, M., Zhu, Y.: Study of Dempster-Shafer theory for image segmentation applications. Image and Vision Computing 20 (2002) 15-23

14. Dempster, A.P.: A generalization of bayesian inference. Journal of the Royal Statistical Society. Series B (Methodological) 30 (1968) 205-247

15. Shafer, G.: A mathematical theory of evidence. Princeton university press (1976)

16. Cremers, D., Rousson, M., Deriche, R.: A review of statistical approaches to level set segmentation: integrating color, texture, motion and shape. International Journal of Computer Vision 72 (2007) 195-215

17. Cremers, D., Schmidt, F.R., Barthel, F.: Shape priors in variational image segmentation: Convexity, lipschitz continuity and globally optimal solutions. In: IEEE Computer Society Conference on Computer Vision and Pattern Recognition. (2008)

18. Paragios, N., Rousson, M., Ramesh, V.: Matching distance functions: A shape-toarea variational approach for global-to-local registration. In: 7th European Conference on Computer Vision. (2002) 775-789

19. Heiler, M., Schnörr, C.: Natural image statistics for natural image segmentation. International Journal of Computer Vision 63 (2005) 5-19

20. Chan, T., Sandberg, B.Y., Vese, L.: Active contours without edges for vectorvalued images. Journal of Visual Communication and Image Representation 11 (2000) 130-141

21. Brox, T., Weickert, J.: A tv flow based local scale estimate and its application to texture discrimination. Journal of Visual Communication and Image Representation 17 (2006) 1053-1073

22. Rousson, M., Paragios, N.: Shape priors for level set representations. In: European Conference on Computer Vision ECCV. Volume 2351 of LNCS., Berlin, Springer (2002) 78-92

23. Martin, D., Fowlkes, C., Tal, D., Malik, J.: A database of human segmented natural images and its application to evaluating segmentation algorithms and measuring ecological statistics. In: Proc. 8th Int'l Conf. Computer Vision. Volume 2. (2001) 416-423

24. Haindl, M., Mikeš, S.: Texture segmentation benchmark. In: Proceedings of the 19th International Conference on Pattern Recognition, ICPR. (2008) 1-4

25. McGuinness, K., O'Connor, N.E.: A comparative evaluation of interactive segmentation algorithms. Pattern Recognition 43 (2010) 434-444 